\title{
FACTORES AMBIENTALES VINCULADOS CON LA APARICIÓN Y DISPERSIÓN DE LAS EPIDEMIAS DE Vibrio EN AMÉRICA DEL SUR
}

\author{
Ronnie G. Gavilán ${ }^{1, a}$, Jaime Martínez-Urtaza1,b
}

\begin{abstract}
RESUMEN
El Vibrio cholerae y el V. parahaemolyticus son las principales especies de Vibrio que ocasionan infecciones en seres humanos. Las infecciones causadas por estos dos patógenos están teniendo una creciente importancia debido a su imparable expansión a nivel mundial. En el presente artículo se resumen los aspectos ecológicos asociados con la llegada y dispersión de las epidemias por $V$. parahaemolyticus y V. cholera en Perú desde una perspectiva sudamericana. De igual forma, se discute las similitudes en la aparición del cólera en 1991 y las infecciones por V. parahaemolyticus en 1997 en Perú, que sirvieron como experimentos únicos para analizar la relación entre las epidemias de Vibrio y los cambios en el medio ambiente. Estas dos radiaciones epidémicas constituyen unos claros ejemplos que apoyan la teoría de la dispersión oceánica de vibrios patógenos y permiten identificar a los episodios de El Niño como un mecanismo potencial de transmisión de enfermedades a través del océano.
\end{abstract}

Palabras Clave: Vibrio Cholerae, Vibrio Parahaemolyticus, Epidemiología Ambiental, Fenómeno de El Niño, Vibriosis (fuente: DeCS BIREME)

\section{ENVIRONMENTAL DRIVERS OF EMERGENCE AND SPREADING OF Vibrio EPIDEMICS IN SOUTH AMERICA}

\begin{abstract}
Vibrio cholerae and V. parahaemolyticus are the two Vibrio species with a major impact on human health. Diseases caused by both pathogens are acquiring increasing relevance due to their expansion at global scale. In this paper, we resume the ecological aspects associated with the arrival and spreading of infections caused by $V$. parahaemolyticus and $V$. cholerae in Peru from a South American perspective. Moreover, we discuss the similarities in the emergence in Peru of cholera cases in 1991 and $V$. parahaemolyticus infections in 1997. These constituted exceptional experiments to evaluate the relationships between the Vibrio epidemics and changes in the environment. The epidemic radiations of $V$. cholerae and $V$. parahaemolyticus constitute to clear examples supporting the oceanic dispersion of pathogenic vibrios and have enabled the identification of El Niño events as a potential mechanism for the spreading of diseases through the ocean.
\end{abstract}

Keywords: Vibrio Cholerae, Vibrio Parahaemolyticus, Epidemiology, El Niño Phenomenon, Vibrio infections (source: MeSH NLM).

\section{INTRODUCCIÓN}

Los vibrios son bacterias que habitan en el medio acuático que incluye especies que ocupan distintos nichos ecológicos dentro de estos ecosistemas (1). EI rango de distribución y las preferencias por un hábitat entre las distintas especies va a estar asociado por las características ambientales dominantes en la zona y, en especial, con los niveles de salinidad y las variaciones de temperatura que van a modular su ciclo de vida en estos sistemas naturales. De todas las especies del género Vibrio, Vibrio cholerae y V. parahaemolyticus son los principales organismos causantes de infecciones en humanos. Las infecciones causadas por estos patógenos tienen una creciente importancia en salud pública debido a que han sufrido un imparable incremento en su incidencia a nivel mundial durante las últimas décadas. Ambas especies son consideradas emergentes $y$ presentan una distribución mundial ${ }^{(2,3)}$.

El Vibrio cholerae está presente de forma natural en ambientes acuáticos de moderada salinidad y es considerado un patógeno facultativo para el hombre. Esta bacteria es la causante del cólera, enfermedad caracterizada por una diarrea acuosa intensa ocasionada por una variante productora de toxina colérica una vez que coloniza el intestino delgado del hombre ${ }^{(4)}$. Estas cepas toxígenas están asociadas principalmente con el serotipo 01 , de distribución mundial y causante de la séptima pandemia de esta enfermedad y con el serotipo

\footnotetext{
Instituto de Acuicultura, Universidad de Santiago de Compostela, Santiago de Compostela, España.

a Biólogo, Magíster en Biología Molecular; ${ }^{\text {b }}$ Doctor en Ciencias Biológicas.
}

$$
\text { Recibido: 01-02-11 Aprobado: 09-03-11 }
$$


0139, que hasta la fecha solo se ha detectado en zonas de sureste asiático ${ }^{(5)}$.

El Vibrio parahaemolyticus es una bacteria marina halófila que se encuentra presente en zonas estuáricas de regiones templadas y tropicales a nivel mundial. Esta bacteria al igual que el $V$. cholerae es considerado un patógeno facultativo que es asociado con el consumo de alimentos marinos crudos. Mientras la mayoría de cepas de $\checkmark$. parahaemolyticus causantes de infecciones muestran la presencia de hemolisinas (tdh y trh), solo una pequeña fracción de las cepas presentes en el medio ambiente marino poseen estos factores de virulencia. El reciente surgimiento de nuevos genotipos patógenos, como es el caso del denominado clon pandémico, que han sufrido un dispersión a nivel global durante los últimos años, ha incrementado la atención de las autoridades sanitarias de diferentes países del mundo y han impulsado una mayor vigilancia de este patógeno ${ }^{(3,6)}$.

Debido a la importancia que tienen estos dos patógenos en la salud pública, en este artículo se resume los recientes avances sobre la influencia de los factores ambientales y patrones oceanográficas en la aparición de los grandes brotes epidémicos de $V$. cholerae y $V$. parahaemolyticus en Sudamérica y particularmente en Perú. De igual forma, se analizan los factores que han podido favorecer el declive de estas enfermedades en la zona, ya que estas condiciones pueden ser claves para entender la dinámica infecciosa de estos patógenos y cómo debemos enfrentarnos a ellos para conseguir contener su expansión.

\section{Los factores ambientales y las infecciones por Vibrio}

La dinámica epidémica de los patógenos asociados con las enfermedades transmitidas por el agua, como es el caso de las infecciones por Vibrio tienen una gran dependencia de los factores físicos-químicos y biológicos del medio ambiente que van a modular su presencia y su abundancia mediante un equilibrio entre crecimiento y depredación. Variaciones en las condiciones ambientales y ecológicas del medio van a repercutir sobre la dinámica biológica de estos microorganismos favoreciendo o limitando su abundancia y promoviendo la aparición de infecciones cuando las condiciones ambientales permiten alcanzar altas cargas de organismos en alimentos marinos de consumo.

Actualmente, se viene estudiando la compleja relación entre las condiciones ecológicas del medio y la dinámica poblacional de estos patógenos, con el fin de poder conocer qué variables son las críticas en el surgimiento de un proceso infectivo o una epidemia. Se ha podido demostrar que cambios en los niveles de temperatura y salinidad del agua de mar tienen una asociación directa con la aparición de brotes infecciosos causados por patógenos de origen acuático en diferentes partes del mundo. La salinidad es un factor crítico que regula la distribución de las distintas especies de Vibrio en función de su afinidad u óptimo osmótico. De esta forma, se conoce que $V$. cholerae tiene afinidad por aguas más salobres que $V$. parahaemolyticus, lo que hace que su distribución a lo largo de las zonas costeras y estuarios esté en función de los gradientes de salinidad existentes ${ }^{(7)}$.

De igual forma, se conoce que la temperatura es un factor fundamental para entender la dinámica poblacional de los vibrios, ya que aumentos estacionales de temperatura del agua de mar inducen de forma directa la proliferación de estos organismos en el medio, alcanzando generalmente mayores densidades en las épocas de mayor temperatura, siempre y cuando los niveles de salinidad en esas épocas sean los óptimos para su expansión demográfica. Estos factores serán fundamentales para entender la dinámica epidémica y el riesgo de infección por Vibrio. V. cholerae y $V$. parahaemolyticus. Estos patógenos son dosisdependientes en su transmisión ambiente-hombre, lo que significa que deben estar presentes en altos niveles en el medio para ser capaces de causar infección. En este sentido, el estudio y la vigilancia de las condiciones ambientales en una zona pueden ser aplicados para inferir el riesgo de la presencia de estos microorganismo $\mathrm{y}$, finalmente, para poder predecir el riesgo de aparición de infecciones en el área. El manejo de esta información y la elaboración de modelos de riesgo es hoy un punto clave en las estrategias para la mitigación de estas enfermedades en varias zonas del mundo ${ }^{(8)}$

Esta estrecha relación, entre el medio ambiente y las epidemias de Vibrio, va a hacer que estos patógenos y sus enfermedades sean uno de los más sensibles a los cambios asociados con el calentamiento global. El patrón de calentamiento observado tiene un impacto directo y de gran magnitud en las zonas costeras, causando un calentamiento de las aguas a nivel local y un descenso de salinidad debido al drástico cambio del régimen de lluvias. Aguas más templadas y menos salinas generadas por un incremento en las lluvias, proporcionará un medio ambiente más propicio para la expansión de las poblaciones de Vibrio en zonas costeras. Pero este fenómeno también permitirá ampliar el rango geográfico de distribución de estos organismos mediante el calentamiento de las aguas de zonas que hasta la fecha permanecían demasiado frías para que existieran niveles de Vibrio suficientes para causar infecciones ${ }^{\left({ }^{8}\right)}$. 
Pero el cambio climático no solo está afectando a las zonas costeras. Se está observado durante los últimos años un cambio en los patrones oceanográficos de circulación oceánica que pueden estar repercutiendo en el clima a nivel global. Las principales corrientes marinas y los patrones de circulación oceánica existentes en los grandes océanos del planeta son actualmente los responsables de la distribución climática tal y como la conocemos en la actualidad. Cualquier mínimo cambio en estos patrones de circulación producirá un serio impacto sobre las zonas afectadas, haciendo variar las condiciones oceanográficas y climáticas en el área. Un claro ejemplo de estas variaciones en los patrones de circulación oceánica es la inversión de las corrientes en la zona ecuatorial durante el fenómeno de El Niño, con su correspondiente impacto biológico en la zona costera del Pacífico de Sudamérica y en el clima a nivel local y mundial, afectando a zonas tan distantes como África, en aspectos a priori no relacionados, como la distribución de vectores de transmisión de enfermedades. Este es el caso del fenómeno de El Niño que recientemente ha sido relacionado directamente con la dispersión y aparición de enfermedades infecciosas como el cólera y la gastroenteritis causada por $V$. parahaemolyticus $^{(7,8)}$ (Figura 1).
De esta manera, cualquier cambio en el equilibrio ecológico del medio marino, bien causado por fenómenos naturales o debidos a la acción del hombre, van a tener un impacto de dimensiones desconocidas en la dinámica poblacional de los vibrios patógenos y en la epidemiología de las enfermedades causadas por estos microorganismos. Conocer las conexiones entre el medio marino y la aparición de enfermedades nos va a permitir predecir el impacto de estos cambios y anticiparnos a la aparición de los casos de infección, minimizando el impacto epidemiológico de estos importantes patógenos humanos en las poblaciones.

\section{PROPAGACIÓN DE LA SÉPTIMA PANDEMIA DEL CÓLERA EN AMÉRICA DEL SUR: LA CONEXIÓN OCEANOGRÁFICA.}

Los casos de cólera que padecen hoy personas de muchos países de casi todos los continentes son parte de la expansión epidémica de la última pandemia de cólera que se inició en Indonesia en 1961 y todavía sigue expandiéndose. Durante los diez primeros años, los brotes de infección estuvieron confinados al continente asiático. En 1970, casos asociados con esta pandemia
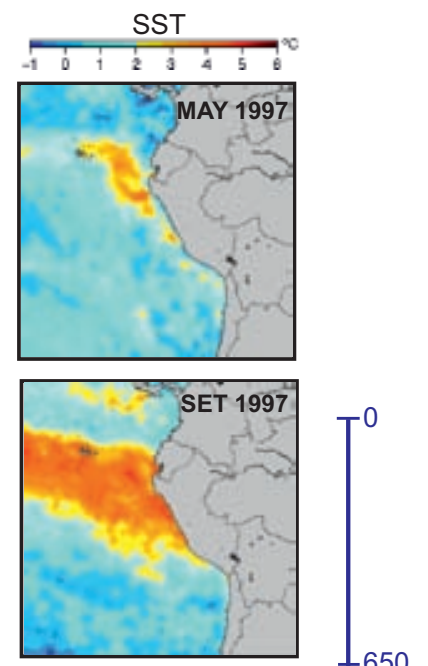

V. parahaemolyticus

V. cholerae
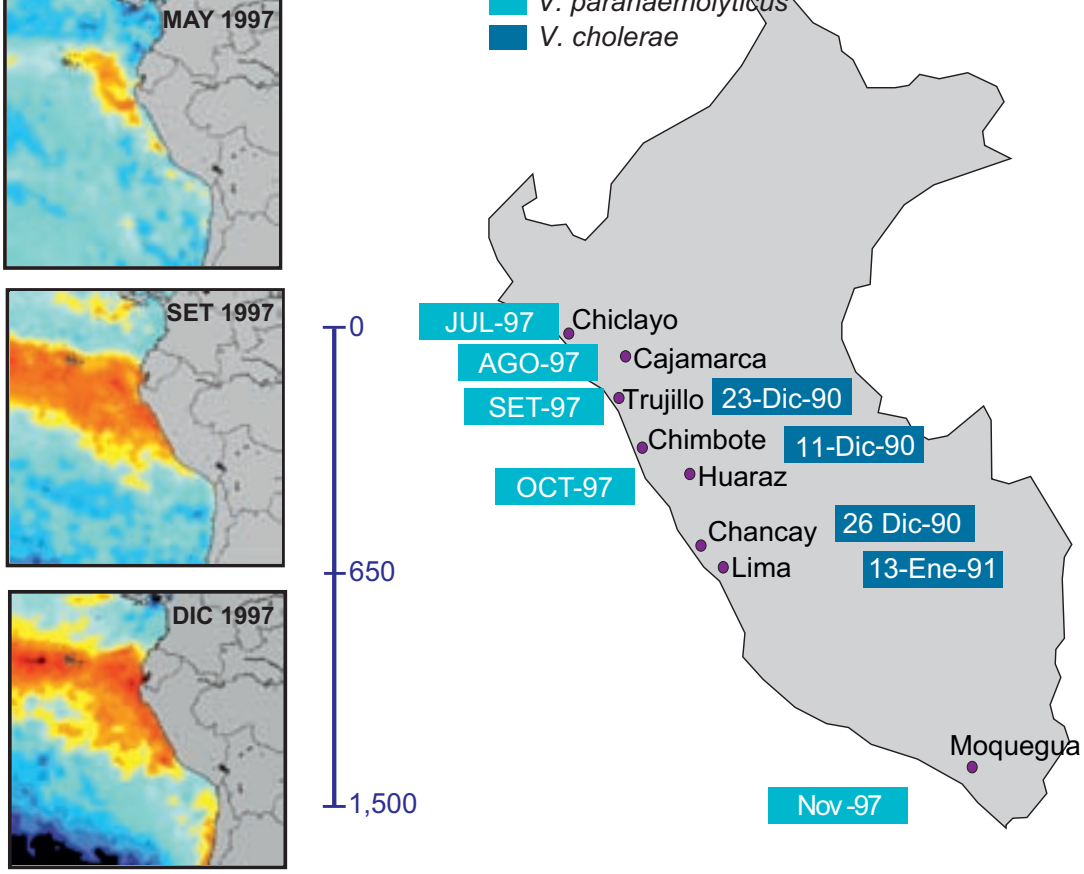

Figura 1. Similitudes entre la aparición y progresión de las epidemias de V. cholera en 1991 y V. parahaemolyticus en 1997 en Perú con la llegada y el movimiento de las aguas de El Niño durante 1997. El movimiento de las masas de agua de El Niño en 1997 fueron graficados utilizando los valores de la temperatura superficial de mar (SST) obtenidos por satélite (Mapas de la izquierda). La dispersión de los brotes de $V$. parahaemolyticus fue representada de color azul y fueron obtenidas a partir de los registros de cepas referidas al INS durante 1997. La detección de los primeros casos del cólera durante la epidemia de 1991 fueron graficados de color rojo de acuerdo con los datos aportados por Seas et al. (11) (Mapa de la derecha). 
fueron detectados en la costa oeste de África, la costa sur de Europa y las islas del oeste del Pacífico. En 1991, casos de cólera se detectaron en la costa de Perú dando inicio a una epidemia de dimensiones dramáticas. Los casos de cólera fueron inicialmente detectados en el norte del país, pero en pocas semanas se extendieron hacía el sur. (Figura 1) En las primeras seis semanas de la epidemia peruana, el Ministerio de Salud del Perú registró 65198 casos de sospecha de cólera y 363 muertes. Desde Perú, la pandemia siguió su expansión y en 1993 ya había alcanzado casi todo el continente americano. El origen y las vías de llegada del agente etiológico causante de la expansión americana de esta pandemia habían permanecido desconocidos.

Para explicar la aparición de este organismo en Perú, se propusieron diferentes mecanismos, entre ellos, la dispersión mediante el agua de lastre de los barcos y la transmisión humana fue la más aceptada, aunque ninguna de estas dos teorías se había podido demostrar fehacientemente. Otra propuesta proponía que el agente infeccioso ya estaba presente en el medio ambiente peruano, y que el calentamiento de las aguas producido por la llegada del fenómeno de El Niño en 1991 provocó el aumento de este organismo en el medio ambiente alcanzando dosis infectivas y causando infecciones por consumo de moluscos o transmitido por el agua ${ }^{(9)}$.

Una hipótesis novedosa fue propuesta por Mouriño-Perez en $1998{ }^{(10)}$, por la cual el movimiento de aguas de Asia a Perú durante el fenómeno de El Niño pudo provocar el transporte de las poblaciones patógenas de $\mathrm{V}$. cholerae que atrapadas en estas aguas llegaron a Perú durante El Niño de 1991, causando el inicio de la epidemia. La revisión de los factores ambientales y la diseminación de infecciones durante la epidemia de 1991 han puesto de nuevo en evidencia la conexión con El Niño. Los primeros casos de cólera se detectaron en el norte del país, y de ahí se propagó norte-sur (11) en paralelo a la llegada y recorrido de las aguas tropicales transportadas por El Niño. (Figura 1) Estos nuevos análisis apoyan la teoría oceánica de la dispersión de las infecciones de cólera que surgieron en Perú en la década de 1990.

\section{DISEMINACIÓN CONTINENTAL DEL CÓLERA Y LA HIPÓTESIS DE LA HIPERINFECTIVIDAD}

Los casos de infecciones de cólera en Perú aparecieron repentinamente en diferentes ciudades costeras $y$, posteriormente, se dispersaron rápidamente, alcanzando en dos semanas a ciudades del interior, llegando a dispersarse por la mayoría del territorio peruano durante el primer mes. La rápida diseminación de $V$. cholerae en áreas continentales de Perú es favorecida por las particulares características biológicas del $\mathrm{V}$. cholerae.
Esta bacteria es no-halófila, es decir, no requiere un ambiente salino para sobrevivir. Esta característica le permite una rápida transmisión a través del agua dulce promoviendo la transmisión mediante los alimentos o agua contaminada. Una de las principales características de las epidemias de cólera es la explosión epidémica: una vez que surgen las infecciones, los casos se propagan de forma explosiva a través de la población en un periodo muy corto. Recientemente, se ha propuesto una explicación a este modelo de transmisión de cólera, de forma que las cepas de $V$. cholerae eliminadas por personas infectadas permanecen en un estado de hiperinfectividad que reduce 700 veces la dosis infectiva para causar la enfermedad, estado que se mantiene durante 18 horas desde la excreción de la bacteria al medio ${ }^{(12)}$. Este modelo propone la importancia relativa de la transmisión fecal-oral en relación a la transmisión ambiente-persona durante la propagación explosiva de los casos de cólera. Según este modelo, las poblaciones patógenas de $\mathrm{V}$. cholerae al llegar a las costas peruanas inicialmente, produjeron pequeños brotes epidémicos por el consumo de productos marinos contaminados con cepas toxígenas (contaminación ambiente-persona). La contaminación de aguas y alimentos con restos fecales de las personas infectadas, promovió la infección de forma directa (humano-humano) mediante cepas en estado infectivo, iniciándose la vertiginosa dispersión de la enfermedad.

\section{DESAPARICIÓN DE LAS INFECCIONES DE CÓLERA}

Desde la aparición del cólera en 1991 las infecciones causadas por $V$. cholerae fueron incrementándose de forma explosiva llegando a producir 322562 casos en ese año; se mantuvieron tasas altas los primeros años y luego fueron disminuyendo progresivamente. En 1998 se observó un rebrote de los casos de cólera que fue asociado con el fenómeno de El Niño que se inició en el invierno de 1997 extendiéndose hasta el verano de 1998 (Figura 2). Estos casos fueron reportados hasta el 2002 cuando se reportaron los últimos casos y hasta la fecha no se han reportado nuevos casos de cólera en Perú (13).

\section{APARICIÓN DEL CLON PANDÉMICO DE Vibrio Parahaemolyticus EN PERÚ}

La aparición de la epidemia de cólera en Perú sensibilizó a las autoridades sanitarias del país en materia de infecciones asociadas a Vibrio. La aparición de un brote epidémico de $V$. parahaemolyticus en el sur de Chile en 2004, causó gran impacto en la comunidad, promoviendo el estudio de la situación epidemiológica de este patógeno en muchas zonas. En este sentido, el Instituto Nacional de Salud de Perú (INS) promovió 
un estudio para conocer la relevancia epidemiológica de este microorganismo en el país.

Durante el transcurso de la epidemia del cólera en Perú en los años 90, se inició la investigación rutinaria de Vibrio en muestras clínicas en el país. La implantación del estudio rutinario de la presencia de vibrios en muestras de heces en hospitales y laboratorios referenciales a lo largo del Perú permitió detectar casos de infección asociados con otra especie de Vibrio, V. parahaemolyticus. Los casos detectados fueron esporádicos, en su mayoría asociados al clon $\mathrm{O} 4: \mathrm{K} 8$, y normalmente debidos al consumo de productos marinos crudos durante los meses de calor. Esta dinámica epidémica cambió abruptamente en 1997, cuando se detectó de forma repentina un gran aumento de casos durante el invierno austral. Este incremento de casos de V. parahaemolyticusfue asociado con la aparición del un clon pandémico asiático de $V$. parahaemolyticus en Perú. El análisis de las cepas y de la dinámica epidémica de los casos de los datos almacenados en el INS, permitió identificar la llegada de este clon al norte del país antes de agosto de 1997, y de ahí se propagó secuencialmente hacia el sur hasta llegar a Lima. Las infecciones estuvieron confinadas a esta zona hasta noviembre, cuando las infecciones fueron extendiéndose en dirección sur alcanzando el departamento de Moquegua y cruzando la frontera con Chile alcanzando Antofagasta (Figura 1). Una vez más, la distribución de los casos y la dinámica de la epidemia coincidían con la detectada en la epidemia de cólera de 1991. El estudio de las condiciones oceanográficas existentes en la costa de Perú durante EI Niño de 1997 evidenció que las aguas cálidas asociadas a este episodio llegaron al norte del país alrededor de mayo de 1997, coincidiendo con la llegada de infecciones, afectando en esta primera fase la zona norte del país. En noviembre de ese año, se detectó una segunda anomalía oceanográfica todavía más fuerte que alcanzó la frontera sur de Perú en noviembre y alcanzó Antofagasta (Figura 1). Una vez más, la aparición de casos y su expansión geográfica a lo largo de la costa de Perú siguió el patrón del movimiento de las masas de agua tropicales transportadas por El Niño ${ }^{(7,8)}$.

Complementariamente, se procedió a analizar y caracterizar las cepas del clon pandémico detectadas en Perú durante 1997 y se pudo mostrar que eran fenotípica y genéticamente indistinguibles a las aparecidas en la India e Indonesia en 1996 que originaron la aparición de la pandemia asociada al clon 03:K6 ${ }^{(7)}$. Paralelamente, un estudio retrospectivo de cepas de $V$. parahaemolyticus obtenidas en varios hospitales de Perú identificó una cepa asilada en 1996 que pertenecía al clon pandémico que era indistinguible genéticamente de las reportadas en Asia, aunque el origen de la infección no pudo ser consistentemente determinado ${ }^{(14)}$.
Este descubrimiento supuso un nuevo apoyo a la teoría de dispersión transoceánica de las infecciones por Vibrio. De acuerdo con nuevas evidencias, las aguas ecuatoriales transportadas por el fenómeno de $\mathrm{El}$ Niño habrían sido las responsables del transporte del clon pandémico de $V$. parahaemolyticus desde Asia a América en 1997, un viaje de más de 14000 km ${ }^{(7)}$. La diseminación de este patógeno estaría relacionada con el transporte de zooplancton por las aguas de El Niño, ya que se ha detectado la llegada de zooplancton tropical en asociación con esta agua cálida. Estas observaciones coinciden con los resultados obtenidos en estudios previos que demostraron que la supervivencia y crecimiento de $V$. parahaemolyticus en el medio marino está estrechamente ligada a su asociación con zooplancton ${ }^{(9,15)}$. El zooplancton le proporcionaría a los vibrios una plataforma para desplazarse por el océano, proveyéndole de protección en este medio ambiente hostil para estos organismos, además de ser una fuente de nutrientes durante el viaje.

\section{DISPERSIÓN OCEÁNICA DE Vibrio Parahaemolyticus EN EL PACIFICO SUDAMERICANO}

La dispersión de las aguas de El Niño de 1997 tuvo su límite al sur en Antofagasta. De forma similar, el brote epidémico de $V$. parahaemolyticus en Perú se extendió hasta Antofagasta, donde los primeros casos fueron detectados en los últimos días de $1997^{(16)}$. Las cepas aisladas en Antofagasta fueron indistinguibles de las de Perú, lo que refuerza su conexión epidémica.

Desde 1997, los casos de V. parahaemolyticus tuvieron un notable retroceso hasta casi desaparecer de todo el país. Sin embargo, en 2004 se produjo un nuevo brote de infección de dimensiones desconocidas hasta la fecha en Sudamérica. Se detectaron más de 3600 casos en el 2004 y 10984 en el $2005^{(17)}$, todos ellos relacionados con el clon pandémico. El origen de estas cepas permaneció desconocido hasta el año pasado, cuando una nueva investigación proporcionó nuevos datos sobre este caso ${ }^{(18)}$. Según los resultados del estudio genético y poblacional de las cepas pandémicas aisladas en Perú y Chile desde 1997, se pudo establecer que en 1997 aparecieron dos subpoblaciones de este clon pandémico en Perú, una de ellas claramente dominante sobre la otra. Esta subpoblación dominante se extendió por toda la costa de Perú y llegó hasta Antofagasta, mientras que la segunda población, muy minoritaria, estuvo confinada a las zonas más al norte de Perú. La retirada de las aguas de El Niño, también originó una regresión de la subpoblación dominante, quedando también restringida a las zonas del norte de Perú. El equilibrio entre estas dos poblaciones cambió a partir del año 2000, cuando la población minoritaria 

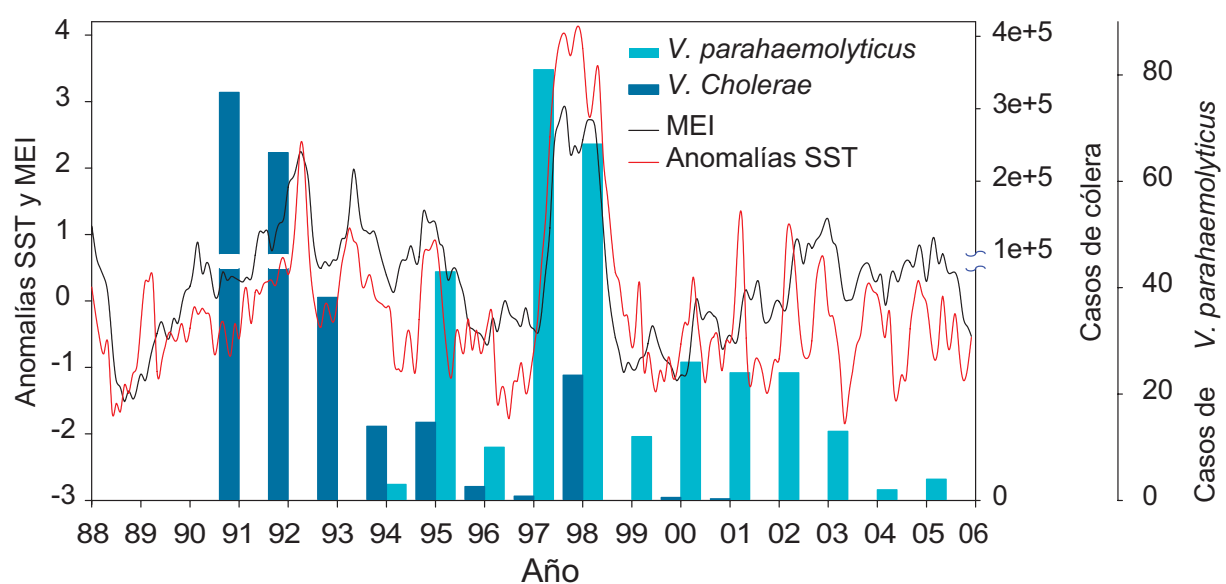

Figura 2. Relación del número de casos de V. cholerae y V. parahaemolyticus durante la aparición de las epidemias de 1991 y 1997 en Perú en relación con dos de los marcadores oceanográficos del fenómeno de El Niño. El número de casos fueron obtenidos de la base de datos del Instituto Nacional de Salud durante el periodo 1991-2006. Los datos de anomalía de la temperatura superficial de mar (SST) y el índice multivariado del ENSO (MEI) fueron obtenidos de la NOAA-CIRES Climate Diagnostic Center website (http://www.cdc.noaa.gov/).

empezó a aumentar su presencia mientras que la otra mostraba un claro declive.

En 2004, cepas identificadas como pertenecientes a la población existente en el norte de Perú aparecieron en el sur de Chile, causando los brotes de infección en Puerto Montt. El análisis de las condiciones oceanográficas dominantes en la zona, previamente a la aparición de los casos, reveló que una anomalía oceanográfica surgió en la zona contrarrestando la corriente de Humboldt dominante en la zona. Esta anomalía desplazó aguas calientes desde el norte de Perú hasta el sur de Chile, y su progresión estuvo correlacionada con la aparición de casos a lo largo del país ${ }^{(18)}$

\section{SITUACIÓN ACTUAL DE LAS INFECCIONES POR VIBRIO PARAHAEMOLYTICUS EN PERÚ}

Posteriormente a la aparición del clon pandémico en Perú, las infecciones causadas por $V$. parahaemolyticus sufrieron un paulatino declive. Durante este periodo, cepas del clon pandémico fueron detectados conjuntamente con cepas de otros serotipos. Esta tendencia continuó hasta el año 2003 , cuando los casos fueron gradualmente disminuyendo hasta retomar los patrones estacionales que existían antes de la llegada del clon pandémico (Figura 2).

Durante el verano de 2009, se detectó un nuevo brote de $V$. parahaemolyticus en el norte del Perú causado por una cepa de $V$. parahaemolyticus con características genéticas distintas al clon pandémico y a las cepas previamente identificadas en Perú. Estas infecciones fueron asociadas al consumo de productos marinos $y$, al igual que otros brotes estacionales anteriores, su incidencia se limitó a la época estival (19).
A pesar del brote epidémico de 2009, la incidencia de infecciones por $V$. parahaemolyticus en Perú ha disminuido considerablemente desde la aparición del clon pandémico, retornando a su dinámica estacional y a cepas principalmente endémicas, como el clon del serotipo $04: \mathrm{K} 8$.

\section{CONCLUSIONES}

Los mecanismos por los cuales se produce la repentina aparición y transmisión explosiva de los patógenos de origen acuático del género Vibrio ha sido un tema sin resolver durante años. Sin embargo, nuevos estudios que integran la investigación epidemiológica, clínica y ambiental, están proporcionando nuevas evidencias que demuestran el papel trascendental que el clima y las condiciones ambientales cumplen en la dispersión de epidemias causadas por Vibrio. De igual forma, la dinámica epidémica asociada con la aparición de infecciones causadas por $V$. cholerae en 1991 y $V$. parahaemolyticus en 1997 en Perú apoya la teoría de dispersión transoceánica de las infecciones por Vibrio. Estudios que se encuentran en curso actualmente buscan identificar los procesos que favorecen estos viajes oceánicos $y$, sobre todo, como pueden estos microorganismos desplazarse y sobrevivir durante largos periodos en un ambiente tan desfavorable como es el mar abierto. En todo caso, estos hallazgos ya se emplean para desarrollar nuevas estrategias para la contención de enfermedades, diseñando herramientas para la predicción del riesgo de presencia de patógenos en tiempo real. De esta forma, será posible anticiparse y establecer medidas para la mitigación del riesgo antes de que las infecciones adquieran una dimensión epidémica, salvaguardando la salud de la población. 


\section{AGRADECIMIENTOS}

Al personal del Laboratorio de Enteropatógenos del Instituto Nacional de Salud de Perú por proporcionar los datos epidemiológicos y cepas investigadas de Perú. Al profesor Romilio Espejo (Universidad de Chile) por el envío de cepas y los datos sobre las infecciones de $V$. parahaemolyticus en Chile. Al profesor Joaquín Triñanes (Universidad de Santiago de Compostela) por suministrar los datos ambientales e imágenes satelitales.

\section{Conflictos de Interés}

Los autores declaran no tener conflictos de interés en la publicación del presente artículo.

\section{REFERENCIAS BIBLIOGRÁFICAS}

1. Thompson F, lida T, Swings J. Biodiversity of vibrios. Microbiol Mol Biol Rev. 2004;68(3):403-31

2. Morris JG. Cholera and other types of vibriosis: a story of human pandemics and oysters on the half shell. Clin Infect Dis. 2003;37(2):272-80.

3. Nair, GB, Ramamurthy T, Bhattacharya SK, Dutta B, Takeda Y, Sack DA. Global dissemination of Vibrio parahaemolyticus serotype O3:K6 and its serovariants. Clin Microbiol Rev. 2007;20(1):39-48.

4. Kaper J, Morris JG, Levine M. Cholera. Clin Microbio Rev. 1995;8(1):48-86.

5. Faruque SM, Albert MJ, Mekalanos JJ. Epidemiology, genetics, and ecology of toxigenic Vibrio cholerae. Microbiol. Microbiol Mol Biol Rev. 1998;62(4):1301-14.

6. Baker-Austin C, Stockley L, Rangdale R, MartinezUrtaza J. Environmental occurrence and clinical impact of Vibrio vulnificus and Vibrio parahaemolyticus: a European perspective. Environmental Microbiology Reports. 2010;2(1):7-18.

7. Martinez-Urtaza J, Huapaya B, Gavilan RG, BlancoAbad V, Ansede-Bermejo J, Cadarso-Suarez C et al. Emergence of Asiatic Vibrio diseases in South America in phase with El Niño. Epidemiology 2008;19(6):829-37.

8. Martinez-Urtaza J, Bowers J, Trinanes J, DePaola A. Climate anomalies and the increasing risk of Vibrio parahaemolyticus and Vibrio vulnificus illnesses. Food Research International. 2010;43(7):1780-90.
9. Colwell R. Global Climate and Infectious Disease: The Cholera Paradigm. Science. 1996; 274(5295):2025-31.

10. Mouriño-Perez RR. Oceanography and the 7th cholera pandemic. Epidemiology. 1998;9(3):355-7.

11. Seas C, Miranda J, Gil Al, Leon-Barúa R, Patz J, Huq A et al. New insights on the emergence of cholera in Latin America during 1991: the Peruvian experience. Am J Trop Med Hyg. 2000; 62(4):513-7.

12. Hartley DM, Morris JG Jr, Smith DL. Hyperinfectivity: A critical element in the ability of $\mathrm{V}$. cholerae to cause epidemics? PLoS Med. 2006; 3(1):e7.

13. Organización Mundial de la Salud. Cholera, 2003. Weekly Epidemiological Record. 2004;79 (31): 281-288.

14. Gil AI, Miranda H, Lanata CF, Prada A, Hall ER, Barreno CM et al. O3:K6 serotype of Vibrio parahaemolyticus identical to the global pandemic clone associated with diarrhea in Peru. Int J Infect Dis. 2007;11(4):324-8.

15. Lipp EK, Huq A, Colwell RR. Effects of global climate on infectious disease: the cholera model. Clin Microbiol Rev. 2002;15(4):757-70.

16. Córdova JL, Astorga J, Silva W, Riquelme C. Characterization by PCR of Vibrio parahaemolyticus isolates collected during the 1997-1998 Chilean outbreak. Biol Res. 2002;35(3-4):433-40.

17. Fuenzalida L, Armijo L, Zabala B, Hernández C, Rioseco ML, Riquelme C. et al. Vibrio parahaemolyticus strains isolated during investigation of the summer 2006 seafood related diarrhea outbreaks in two regions of Chile. Int $J$ Food Microbiol. 2007;117(3):270-5.

18. Ansede-Bermejo J, Gavilan RG, Triñanes J, Espejo RT, Martinez-Urtaza J. Origins and colonization history of pandemic Vibrio parahaemolyticus in South America. Mol Ecol 2010;19(18):3924-37.

19. Dirección General de Epidemiología - Ministerio de Salud. Brotes y otras emergencias sanitarias SE 10. Boletín Epidemiológico. 2009;18(9):171-2.

Correspondencia: Dr. Jaime Martinez-Urtaza

Dirección: Instituto de Acuicultura, Universidad de Santiago de Compostela Campus Sur S/N, 15782, Santiago de Compostela, España.

Teléfono: +34881816060 / Fax: +34981547165

Correo electrónico: jaime.martinez.urtaza@usc.es 